\title{
Regulation of dynamin-related protein 1 (DRP1) levels modulates myoblast atrophy induced by C26 colon cancer-conditioned medium
}

\author{
Xiangyu Mao', Qingyang Meng ${ }^{1}$, Jun Han ${ }^{1}$, Lei Shen ${ }^{1}$, Xiangyu Sui ${ }^{1}$, Yihua Gu ${ }^{2}$, Guohao Wu ${ }^{1}$ \\ ${ }^{1}$ Department of General Surgery, Zhongshan Hospital of Fudan University, Shanghai, China; ${ }^{2}$ Key Laboratory of Contraceptive Drugs and Devices, \\ Shanghai Institute of Planned Parenthood Research, Shanghai, China \\ Contributions: (I) Conception and design: G Wu, Q Meng, X Mao; (II) Administrative support: Q Meng, Y Gu; (III) Provision of study materials \\ or patients: Y Gu, X Mao, J Han, L Shen, X Sui; (IV) Collection and assembly of data: X Mao; (V) Data analysis and interpretation: X Mao; (VI) \\ Manuscript writing: All authors; (VII) Final approval of manuscript: All authors. \\ Correspondence to: Guohao Wu. Department of General Surgery, Zhongshan Hospital of Fudan University, Shanghai, China. Email: prowugh@163.com.
}

Background: Cancer associated-cachexia, which involves progressive skeletal muscle loss, is induced by multiple factors. However, the underlying mechanism remains unclear. Dynamin-related protein 1 (DRP1), a major modulator of mitochondrial fission, has been reported to participate in muscle turnover. This study aimed to explore the role of DRP1 in muscle during the process of cancer associated-cachexia (CAC) via an in vitro model and the mechanisms involved.

Methods: C26 colon cancer cell-conditioned medium (CM) was used to incubate with C2C12 myotubes to simulate cachexia. Myotubes were then transduced with lentiviral vectors of DRP1-small interfering RNA (siRNA), DRP1 overexpression plasmid, or a control plasmid to regulate the DRP1 levels, and their diameters were assessed using a biological microscope. Furthermore, transcriptome sequencing was performed to screen the pathways involved, and real-time polymerase chain reaction (RT-PCR) was used for verification.

Results: The cachexia model was successfully established with a decreased myotube diameter and increased DRP1 expression. DRP1 knockdown significantly ameliorated myotube wasting during cachexia, while DRP1 overexpression intensified this phenomenon. Transcriptome sequencing indicated that DRP1 knockdown was associated with the activation of ribosomal biogenesis. However, PCR results showed that compared to the control, one of the ribosomal biogenesis marker's (Ubf) level was decreased by C26 CM, and DRP1 knockdown did not significantly restore its level.

Conclusions: During C26 CM-induced cachexia, DRP1 was activated, while the regulation of DRP1 levels was able to modulate the atrophy of C2C12 myotubes. The underlying mechanism of the alleviated muscle atrophy induced by DRP1 knockdown was likely associated with increased ribosomal activity.

Keywords: Cachexia; neoplasms; muscular atrophy; dynamins; ribosomes

Submitted Apr 14, 2021. Accepted for publication Jun 15, 2021.

doi: $10.21037 /$ tcr-21-751

View this article at: https://dx.doi.org/10.21037/tcr-21-751

\section{Introduction}

Cancer associated-cachexia (CAC) is a syndrome that involves unintentional weight loss and metabolic disorder of proteins. It induces organic functional impairment, increases cancer treatment complications, affects quality of life, and ultimately causes death, all of which result in devastating effects on patients. Neoplasms of digestive system (such as pancreatic, gastro-oesophageal, colorectal) have a relative high rate $(45-70 \%)$ to develop CAC $(1,2)$. Cachexia is driven by a combination of reduced nutrients 
intake and metabolic changes. These metabolic changes include increased energy expenditure, elevated catabolism and inflammation. Different from simple starvation and malnutrition, cachexia could not be reversed by supplement of adequate nutrients. Up to now, no drug is approved to this syndrome (3). Skeletal muscle atrophy is emerged as a pivotal character of CAC, as from its definition (ongoing loss of skeletal muscle mass, with or without loss of fat mass) (4). Also, it is an independent prognostic factor in patients with CAC (5).

Mitochondria, the central organelles of energy production in skeletal muscle, are constantly under states of fusion and fission. With fusion they could generate an elongated mitochondrion by joining two mitochondria to expand their interconnectivity and network. This process also removes and transforms damaged and dysfunctional components into healthy mitochondria. In contrast, the fission process of mitochondria separates damaged and dysfunctional components from mitochondria to prepare them for autophagy (6). These processes are essential for the maintenance of mitochondrial quality, function, and morphology (7). Dynamin-related protein 1 (DRP1), a member of the dynamin superfamily of guanosine triphosphatase (GTPases), is the main factor modulating mitochondrial fission in mammalian cells (8). It gathers at the outer membrane of mitochondrion and forms a polymeric ring compound at the GTPdependent mitochondrial fission site. It then interacts with other proteins inserted in the outer membrane, including mitochondrial fission factor (MFF) and fission protein 1 (FIS1), to activate fission. On the other hand, the fusion process of the outer and inner membrane is primarily regulated by mitofusin 1 (MFN1), mitofusin 2 (MFN2), and optic atrophy protein 1 (OPA1) (9).

Recent studies have shown that mitochondrial dynamics play a crucial role in muscle turnover. In a mouse model of fasting, Romanello et al. revealed that inhibiting FIS1 expression in skeletal muscle attenuated mitochondrial fission and protected the muscle from wasting, whereas overexpressing FIS1 decreased the myofiber size (10). Similar outcomes were obtained in CAC. White et al. demonstrated that FIS1 was upregulated and MFN1/MFN2 were downregulated in an Interleukin-6 (IL-6)-dependent cachexia model of $\mathrm{Apc}^{\mathrm{Min} / \mathrm{t}}$ mouse ( $\mathrm{Apc}^{\mathrm{Min}}$ is a point mutation in the murine homolog of the APC gene (Min, multiple intestinal neoplasia)), as an anti-IL-6 receptor antibody and exercise attenuated this phenomenon $(11,12)$. Meanwhile, the study by Marzetti et al. of older-aged gastric cancer patients indicated that FIS1 mRNA was upregulated in individuals with CAC compared to those without (13). On the other hand, Sebastian et al. reported that skeletal muscle-specific MFN2 knockout in mice induced muscle atrophy (14). Altogether, these results indicated that muscle atrophy was apparently associated with the status of enhanced fission and repressed fusion.

However, recent studies have drawn an opposing conclusion that cachexia could impair both fusion and fission processes. The results of Barreto et al. from two mouse models of cachexia (chemotherapy- and colon tumorinduced) indicated that mitochondrial fission and fusion were suppressed, which manifested as a downregulated expression of MFN2/OPA1 and DRP1 proteins (15). Furthermore, Brown et al.'s study in a Lewis lung tumor mouse model implicated a downregulated DRP1 expression (16).

Considering the intimate relationship between mitochondrial fission and muscle turnover, as well as the potentially conflicting results of existing studies, it is important to explore the definite role of DRP1, the major regulator of fission, during $\mathrm{CAC}$, in detail.

In this study, we aimed to explore the role of DRP1 in muscle by up- and down-regulating its levels in an in vitro model of cachexia. We also intended to discuss the possible mechanism involved in the regulation process. We present the following article in accordance with the MDAR reporting checklist (available at https://dx.doi.org/10.21037/ tcr-21-751).

\section{Methods}

\section{Cell culture and medium collection}

C26 BALB/c mouse colon adenocarcinoma cells and C2C12 C3H mouse myoblasts (CRL-2638 and CRL1772, respectively; American Type Culture Collection, Manassas, VA, USA) were obtained from Dr. Han (Zhongshan Hospital, Shanghai, China). The cells were cultured in high-glucose Dulbecco's modified Eagle's medium (DMEM), with penicillin (100 units/mL), streptomycin $(100 \mu \mathrm{g} / \mathrm{mL})$ (KeyGen Biotech, Jiangsu, China), and 10\% fetal bovine serum (FBS; CellSera, Rutherford, Australia). C2C12 myoblasts were cultured until they reached $\geq 95 \%$ confluence, and were then placed in DMEM with 2\% horse serum (HS; Gibco, Grand Island, NY, USA) for differentiation. This process typically took $96 \mathrm{~h}$.

To obtain C26 conditioned medium (CM), cancer 
cells were cultured in 10\% FBS DMEM to reach $80 \%$ confluence, and were subsequently placed with $2 \%$ HS DMEM for an additional $24 \mathrm{~h}$. After centrifugation at $1,000 \times \mathrm{g}$ for $5 \mathrm{~min}$, we collected the supernatants and diluted them with $2 \%$ HS DMEM at a concentration of $33 \%$ as $\mathrm{C} 26 \mathrm{CM}$, and the control medium was DMEM with $2 \%$ HS.

\section{Construction of lentiviral vectors modulating DRP1 expression and cellular transduction}

To understand the role of DRP1 in our model, we obtained vectors with siRNAs targeting DRP1 and a negative control siRNA from GeneChem Inc. (Shanghai, China). Briefly, the protocol was as follows: firstly, three siRNAs of the target sequence and a control siRNA were designed. After synthesizing polymerase chain reaction (PCR) products based on these designs, they were cloned into the central part of a GV248 $(11.5 \mathrm{~kb})$ lentiviral vector. The relevant siRNA, enhanced green fluorescent protein (eGFP), as well as puromycin resistance gene were expressed in GV248. Next, vectors were transformed into Escherichia coli (DH5a) and the positive transformants were identified by PCR. We then used the X-tremeGENE reagent (Roche, Basel, Switzerland) to transfect $293 \mathrm{~T}$ cells with the plasmid mixtures (GV248 with auxiliary packaging plasmids Helper 1.0 and Helper 2.0, at a ratio of Helper 1: Helper 2: $G V=15: 10: 20 \mu \mathrm{g}$ ) for $48 \mathrm{~h}$. The lentiviruses were then harvested with a collection of the supernatants, followed by concentrations and viral titers measurement. Furthermore, a GV358 lentiviral vector with a DRP1 expression plasmid was constructed with a similar protocol.

For the process of transduction, myoblasts were infected with lentiviruses at a multiplicity of infection (MOI) of 100 for $12 \mathrm{~h}$ in the presence of $2 \%$ HS DMEM. After culturing for 3-4 days, we observed the expression of eGFP using a fluorescence microscopy (Ti-E+A1R+STORM; Nikon, Tokyo, Japan) to evaluate the efficiency of transduction, and cell lines with an appropriate efficiency $(>80 \%)$ were applied. Next, we verified their expression of DRP1 by western blotting. Afterwards, we performed a puromycin selection (concentrations of 0.1/0.3/0.5/0.7/0.9/1.1/1.3/1.5/ $1.7 / 1.9 \mu \mathrm{g} / \mathrm{mL}$ were examined, and $0.9 \mu \mathrm{g} / \mathrm{mL}$ was selected as optimal) to generate a cell line with stable transduction of lentivirus, according to the manufacturer's protocol. Notably, given that excessive DRP1 knockdown in mouse embryos is lethal $(17,18)$ and may have a repressive effect on $\mathrm{C} 2 \mathrm{C} 12$ differentiation (19), we proposed to select the cell line with an appropriate efficiency of knockdown (nearly 50-70\%).

\section{Measurement of myotube diameter}

After differentiation, the myotubes were divided into five groups: myotubes of DRP1 knockdown cultured with CM (KD), myotubes of DRP1 overexpression cultured with CM (OE), myotubes of DRP1 control (transduced with empty lentivirus vector) cultured with $\mathrm{CM}$ (Dc), myotubes of non-virus exposed to CM (CM) (treated with $33 \%$ $\mathrm{C} 26 \mathrm{CM}$ for $48 \mathrm{~h}$ ), and myotubes of non-virus exposed to control medium (2\% HS DMEM for 48 h) (HS). For all conditions, the medium was renewed every $24 \mathrm{~h}$. Myotubes were observed and photographed at $\times 200$ magnification under a biological microscope (Nikon ECLIPSE TS100-F, Japan). The myotube diameter was determined by an average measurement obtained from three different points randomly distributed along the entire myotube. We used ImageJ software (National Institutes of Health, Bethesda, $\mathrm{MD}$, USA) to randomly measure $\geq 30$ myotubes from $6-8$ fields for each group. Every group was repeated three times with a similar result.

\section{Western blotting}

The myotubes were harvested and lysed with radioimmunoprecipitation assay (RIPA) lysis buffer (Biotechwell, Shanghai, China) at $4{ }^{\circ} \mathrm{C}$ for $15 \mathrm{~min}$, and were centrifuged at 12,000 $\times g$ for $30 \mathrm{~min}$. The supernatants were collected and boiled in sodium dodecyl sulfate (SDS) loading buffer, and stored at $-80{ }^{\circ} \mathrm{C}$ until use. Thereafter, the proteins were separated by SDS-polyacrylamide gel electrophoresis (PAGE) and then transferred to nitrocellulose membranes. The membranes were blocked with $5 \%$ milk diluted with Tris-buffered saline with Tween-20 (TBST) for $1 \mathrm{~h}$, and then incubated with primary anti-DRP1 antibody (1:750; \# 40853; Sab, College Park, Maryland, USA), or anti-glyceraldehyde 3-phosphate dehydrogenase (GAPDH) antibody (1:10,000; \# AC001; Abclonal, Wuhan, China) at $4{ }^{\circ} \mathrm{C}$ overnight. Subsequently, the membranes were exposed to secondary antibody for 1 h. Enhanced chemiluminescence (Tanon, Shanghai, China) was used to visualize the bands and the images were scanned and photographed by an automated image analysis system (5200s, Tanon, Shanghai, China). The bands were subsequently quantified using ImageJ (National Institutes of Health, Bethesda, MD, USA). Every group was repeated 
Table 1 Gene-specific primers for real-time PCR

\begin{tabular}{lll}
\hline Gene & Forward primer $\left(5^{\prime} \rightarrow 3^{\prime}\right)$ & Reverse primer $\left(5^{\prime} \rightarrow 3^{\prime}\right)$ \\
\hline C-Myc & CCTTCTCTCCTTCCTCGGACT & TGCCTCTTCTCCACAGACACC \\
UBF & CCTGACCTTATCCAGAATGCCAA & TAATCTCATCCGGCCGCAC \\
$\mathrm{Nop56}$ & CACCTTCATTGGCCGAGCAG & CTCATAGAAAGACAGCCGTTCC \\
$\mathrm{Ncl}$ & TTGTACGTGCTCCAGAGTCG & GTTTGCCAGCCTTTGCGA \\
$\mathrm{Pol}-\mathrm{I}$ & CTTACCGACGGGGGAAGATG & ATCTGTGAGGAACCCTTGCTG \\
GAPDH & CCTCGTCCCGTAGACAAAATG & TGAGGTCAATGAAGGGGTCGT
\end{tabular}

Pol-I, RNA polymerase I; c-Myc, MYC proto-oncogene; Ubf, upstream binding factor; Nop56, nucleolar protein 56; Ncl, nucleolin; GAPDH, glyceraldehyde 3-phosphate dehydrogenase; PCR, polymerase chain reaction.

three times with a similar result.

\section{Transcriptome sequencing}

We selected samples from the KD, Dc, and CM groups to perform transcriptome sequencing analysis. The myotubes were harvested and dissolved in Trizol (Thermo Fisher Scientific, Waltham, MA, USA) treatment according to the manufacturer's protocol to extract total RNA. After quantifying with NanoDrop (Thermo Fisher Scientific, Waltham, MA, USA) and qualifying with Agilent 2100 Bioanalyzer (Agilent Technologies, Palo Alto, CA, USA), the value of RNA integrity number (RIN), which reflects the quality of RNA, was guaranteed $>7$. One $\mu \mathrm{g}$ RNA was utilized for library preparation. The processes of sequencing and analysis were carried out by GENEWIZ (Suzhou, China), and sequencing was conducted on an Illumina HiSeq instrument (Illumina, San Diego, CA, USA). Raw data were processed by Trimmomatic (v0.30) to guarantee high quality. DESeq (v1.6.3) was used to analyze the differentially expressed genes between two groups (KD vs. Dc; KD vs. CM; Dc vs. CM), based on a model of negative binomial distribution. Enriched genes with a $\mathrm{P}$ value $<0.05$ were identified and assigned to the associated Gene Ontology (GO) terms. Clustering analysis was performed to classify the data by calculating its similarity. This process grouped genes exhibiting similar expression patterns and therefore recognized the function of unknown genes to deduce whether they act in the same metabolic process or pathway. The Kyoto Encyclopedia of Genes and Genomes (KEGG) is a collection of databases dealing with genomes, biological pathways, diseases, drugs, and chemical substances (http://en.wikipedia.org/wiki/KEGG). We used in-house scripts to enrich the significant differentially expressed genes in the KEGG pathways.

\section{Real-time PCR}

The procedure for extracting and preparing total RNA was mentioned above, and the specific primers for the markers of ribosomal biogenesis are presented in Table 1 . Subsequently, we used $2 \mu \mathrm{g}$ RNA for reverse transcription. The process of quantitative real-time PCR was performed in a 7900HT PCR system (Applied Biosystems, Foster City, CA, USA) with a FastStart Essential DNA Green Master (Roche, Basel, Switzerland). GAPDH was used as a housekeeping gene for the normalization of mRNA levels, and the $2^{-\triangle \Delta \mathrm{CT}}$ method was used for the relative expression of genes. Every group was repeated three times with a similar result.

\section{Statistical analysis}

All data were analyzed using a GraphPad Prism software (version 8.02; San Diego, CA, USA), and the results were displayed as mean \pm standard deviation. $\mathrm{P}<0.05$ was considered statistically significant. One-way analysis of variance (ANOVA) was used to determine the differences between groups. Post-hoc tests were performed using the Bonferroni method.

\section{Results}

\section{Model of muscle atrophy induced by C26 CM with C2C12 myotubes}

As C26-mediated atrophy has been widely used during cachexia in vitro research (20-22), we firstly assessed whether C26 CM could cause atrophy. The outcomes 
A
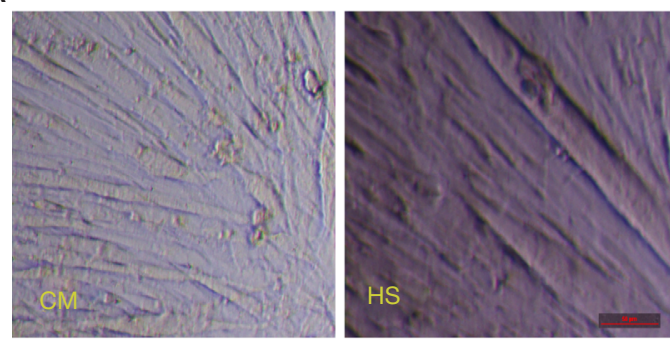

B

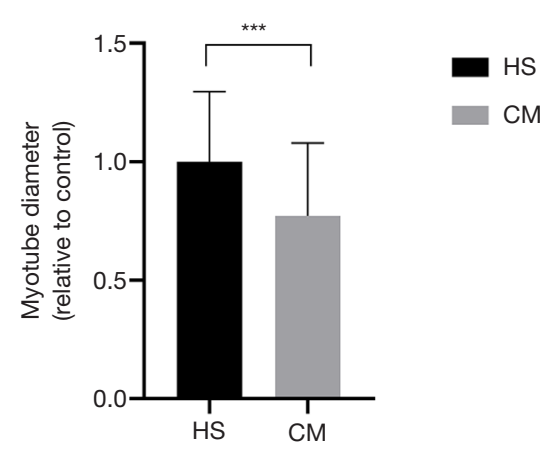

C
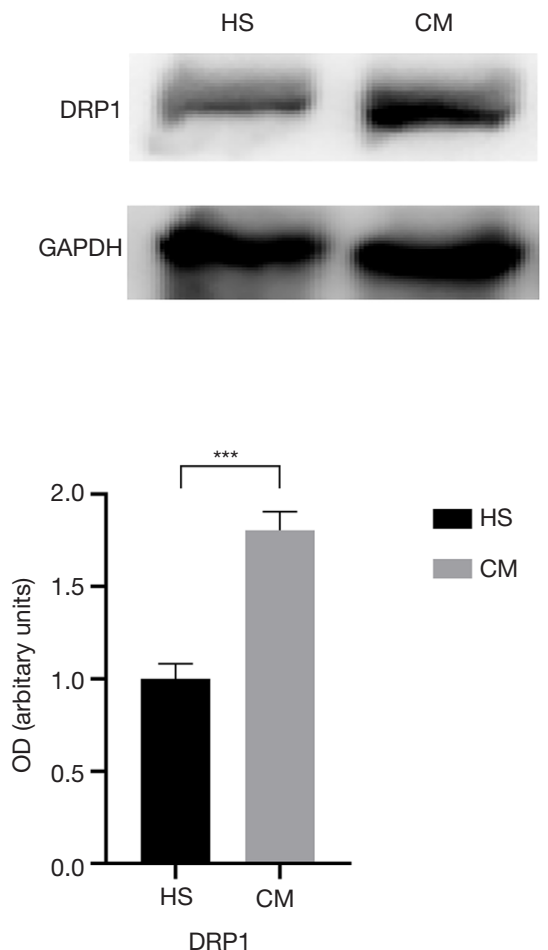

Figure 1 C26 CM induced myotube atrophy and activated DRP1 expression. (A) C2C12 myotubes visualized by a biologic microscopy at $\times 200$ magnification and. (B) Analysis of myotubes' mean diameters. (C) DRP1 protein levels of the HS/CM group by western blotting. Data were shown as mean $\pm \mathrm{SD}$. ${ }^{* *} \mathrm{P}<0.01$. HS, myotube cultured in DMEM of $2 \%$ HS (horse serum); CM, myotube cultured in DMEM of $33 \%$ C26 CM (conditional medium); DRP1, dynamin-related protein 1; GAPDH, glyceraldehyde 3-phosphate dehydrogenase.

exhibited that culturing in $\mathrm{C} 26 \mathrm{CM}$ reduced the diameter of myotubes (Figure 1A). Specifically, the reduction of mean diameter was $23 \%$ compared with HS group $(\mathrm{P}=0.006)$ (Figure $1 B$ ). Meanwhile, an increase of DRP1 protein expression $(\mathrm{P}=0.0093)$ was observed (Figure 1C). In early differentiation stage of $\mathrm{C} 2 \mathrm{C} 12$ myoblasts, an upregulated mitochondrial fission and DRP1 is accompany with an activated autophagy (usually D1). Afterwards, both autophagy and fission are suppressed (usually from D2 and on) $(19,23)$. Therefore, the expression of DRP1 in myotube is not high. Our results indicated that C26 CM induced atrophy and DRP1 activation in C2C12 myotubes.

\section{Regulation of DPR1 modulates atrophy in C2C12 myotubes}

Since our initial results indicated an association between enhanced DRP1 expression and C26 CM-induced atrophy, we continued to explore whether enhanced or reduced
DRP1 levels would influence the anti-atrophic ability of C26 CM. After a successful construction of lentiviral vectors with DRP1-siRNA, DRP1 overexpression plasmid and control plasmid, myoblasts were transduced with the vectors. The fluorescence of eGFP guaranteed a transduction efficiency of $>80 \%$ for all vectors (Figure 2A). Next, western blotting confirmed the expression of DRP1 in each group (KD/Dc $\mathrm{P}=0.0006$; OE/ $\mathrm{Dc} \mathrm{P}=0.0026$ ) (Figure $2 B$ ). We chose a reduction of $68 \%$ for the KD group out of three siRNA samples. Of note, neither DRP1 overexpression nor DRP1 knockdown inhibited the differentiation of myoblasts.

Interestingly, after adding the C26 CM, we observed a direct visualization that the myotubes of the OE group appeared to be prominently thinner compared with those of the Dc and CM groups. Meanwhile, the myotubes from the KD group displayed a markedly thicker morphology compared to the other groups (Figure 2C). This was also confirmed via the measurement of mean diameters (OE to 

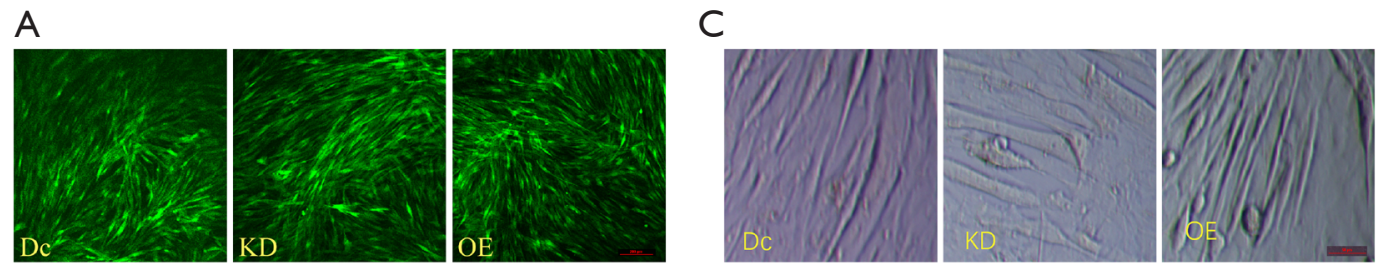

B

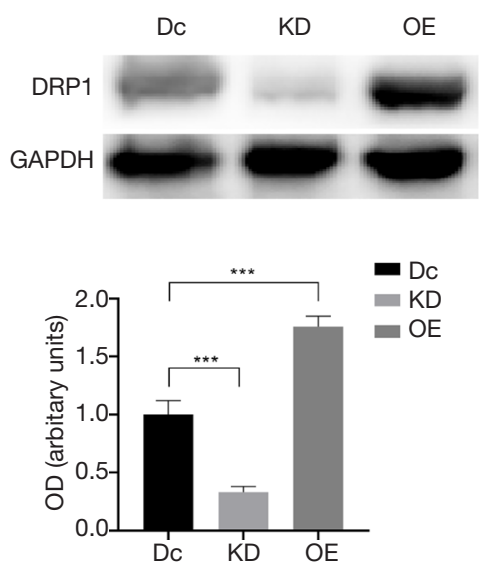

D

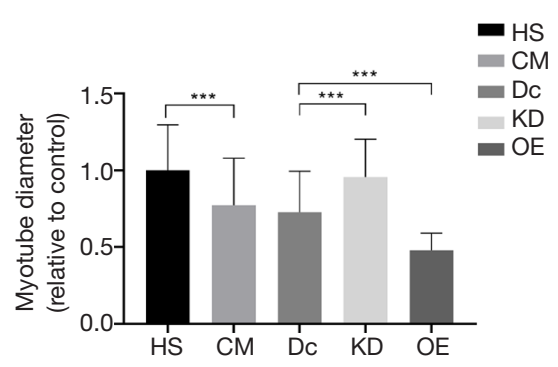

Figure 2 Regulation of DRP1 levels modulates myotube wasting during C26 CM-induced muscle atrophy. (A) Fluorescence images of eGFP expression and. (B) DRP1 protein levels of KD/OE/Dc group. (C) C2C12 myotubes visualized by a biologic microscopy and. (D) Analysis of myotubes' mean diameters of KD/OE/Dc group. Data were shown as mean $\pm \mathrm{SD}$. ${ }^{* *} \mathrm{P}<0.01$ vs. control. eGFP, enhanced green fluorescent protein. HS, myotube cultured in DMEM of 2\% HS (horse serum); CM, myotube cultured in DMEM of $33 \%$ C26 CM (conditional medium); Dc, myotube infected with control lentivirus in CM; OE, myotube infected with overexpressed lentivirus in CM; KD, myotube infected with knockdown lentivirus in CM; DRP1, dynamin-related protein 1; GAPDH, glyceraldehyde 3-phosphate dehydrogenase.

Dc/CM: $\mathrm{P}=0.0008 / \mathrm{P}<0.0001 ; \mathrm{KD}$ to $\mathrm{Dc} / \mathrm{CM}: \mathrm{P}=0.0054 /$ $\mathrm{P}=0.0429$ ) (Figure 2D). This outcome demonstrated that during C26 CM-induced cachexia, DRP1 overexpression in C2C12 myotubes increased myotube atrophy, whereas DRP1 knockdown attenuated the correlated muscle wasting.

\section{Transcriptome sequencing revealed that DRP1 knockdown- induced atrophy alleviation involved activated ribosomal biogenesis}

Since DRP1 knockdown significantly reduced muscle atrophy, we further explored its overall impact by transcriptome sequencing. Comparing the KD and Dc groups, a total of 1,397 genes were down-regulated and 1,532 genes were up-regulated among the differentially expressed genes, as shown in the MA plot [a plot of logintensity ratios (M-values) vs. log-intensity averages (A-values)] (Figure 3A). Additionally, the outcomes of clustering analysis from differentially expressed genes were exhibited as a heatmap (Figure 3B). Notably, the genetic distribution pattern of the CM group was similar to that of the Dc group, and the analysis between the Dc and CM groups uncovered two genes that were upregulated and none were down-regulated (data not shown). This discrepancy indicated that lentivirus transduction itself had a minor influence on myotubes. Furthermore, the KEGG analysis indicated that the main pathways, including ribosome, oxidative phosphorylation, Parkinson's disease, Alzheimer's disease, and Huntington's disease, were involved. These significantly altered pathways were displayed as a scatter plot (Figure $3 C$ ). It is worth noting that the genes related to ribosome proteins and oxidative phosphorylation were remarkably enhanced compared to the KD and Dc groups (Figure 3D,E). Thus, the transcriptome analysis provided an insight into the molecular mechanisms of DRP1 knockdown-alleviated atrophy, which specifically involved ribosomal biogenesis 

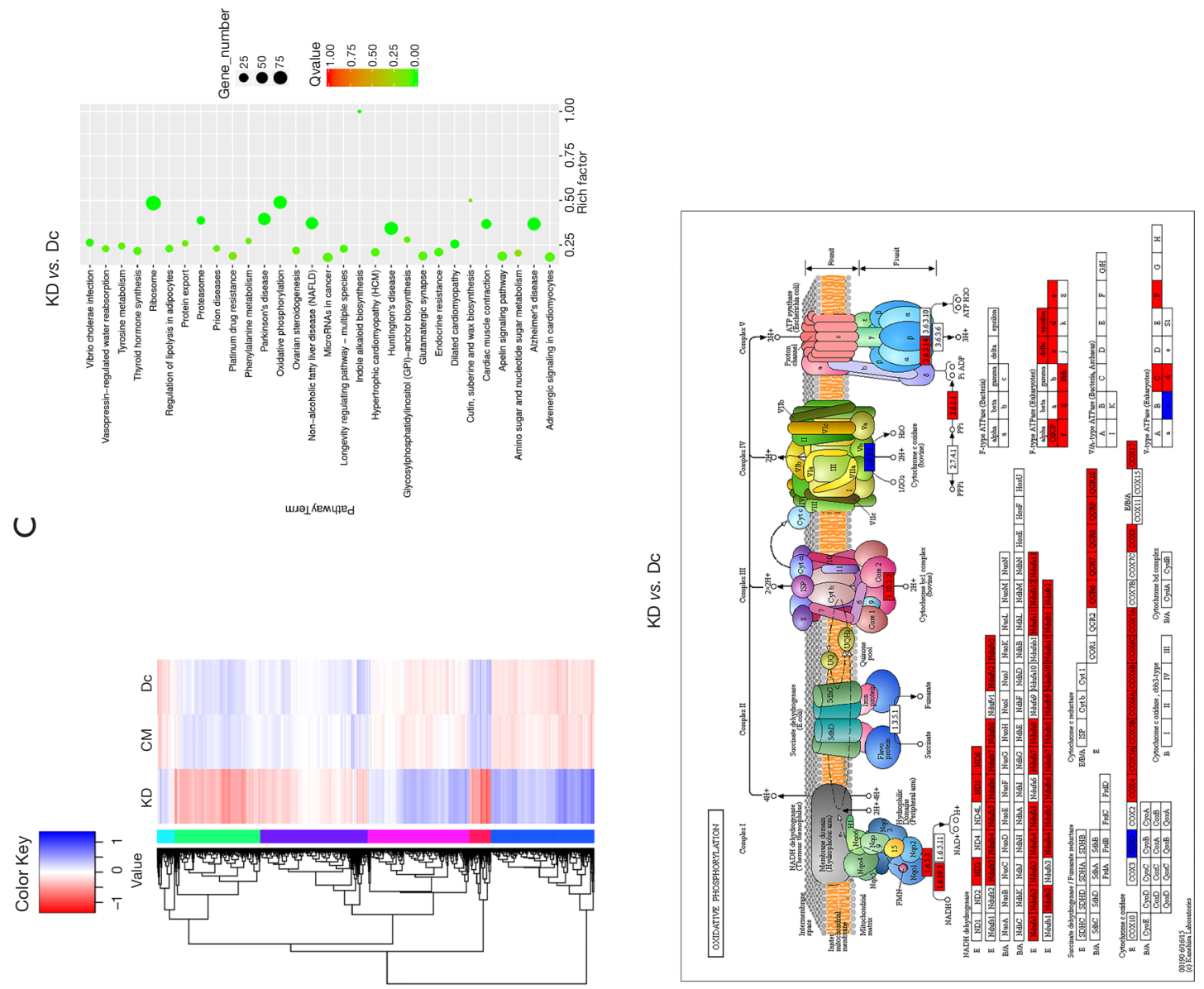

$\infty$

ш
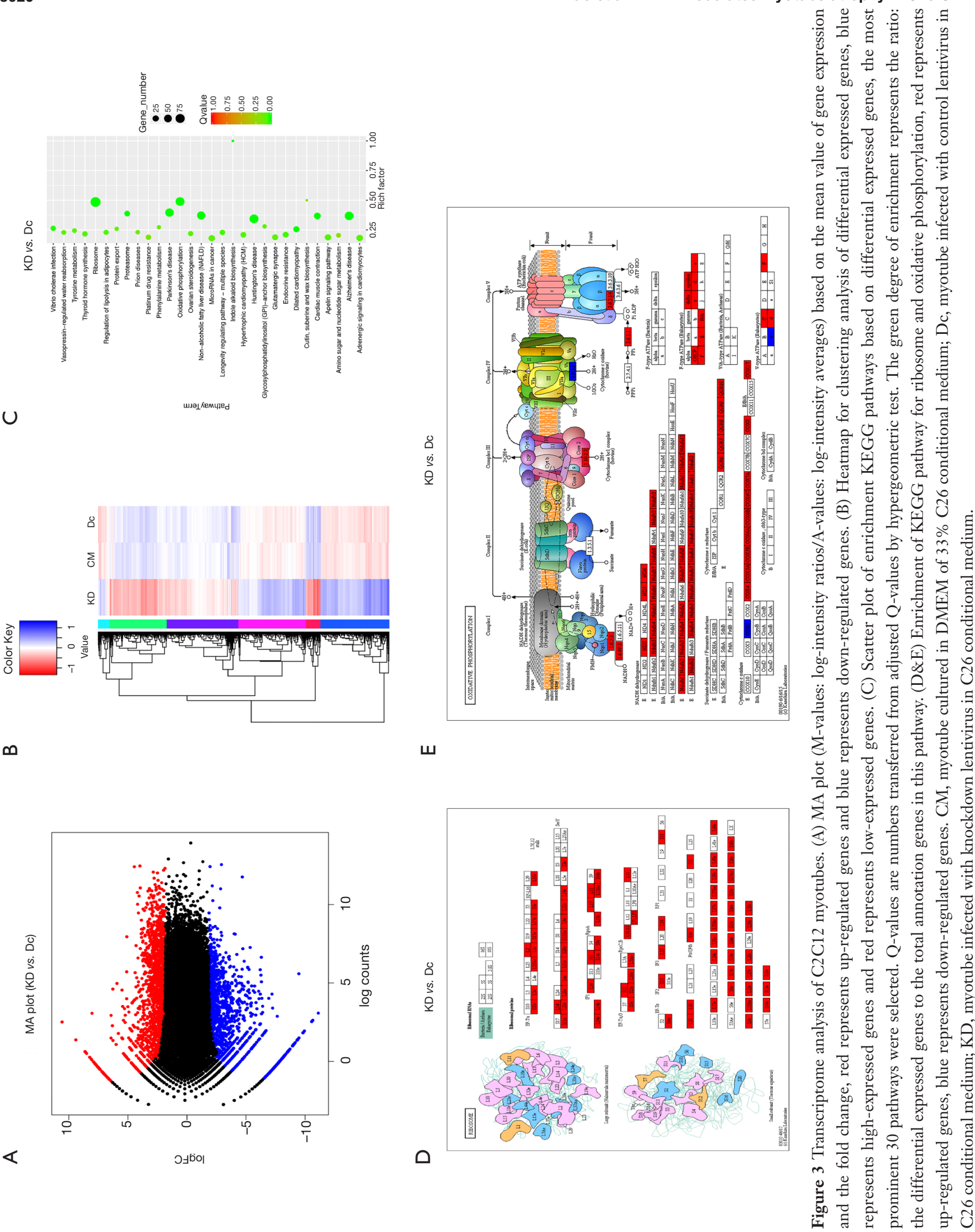


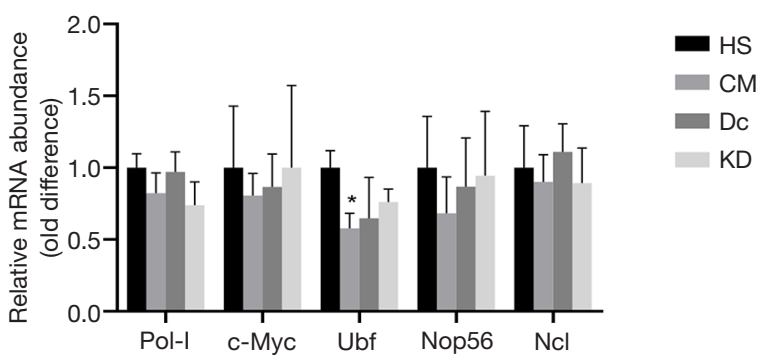

Figure 4 C26 CM reduced the marker of ribosomal biogenesis at mRNA levels. Gene expression levels of Pol-I, c-Myc, Ubf, Nop56, Ncl from C2C12 myotubes in KD/Dc/CM/HS groups using a real-time PCR method $(n=4)$. Data were shown as mean \pm SD. ${ }^{*} \mathrm{P}<0.05$. HS, myotube cultured in DMEM of $2 \%$ HS (horse serum); CM, myotube cultured in DMEM of 33\% C26 conditional medium; Dc, myotube infected with control lentivirus in C26 conditional medium; KD, myotube infected with knockdown lentivirus in C26 conditional medium; Pol-I, RNA polymerase I; c-Myc, MYC proto-oncogene; Ubf, upstream binding factor; Nop56, nucleolar protein 56; Ncl, nucleolin; PCR, polymerase chain reaction.

and oxidative phosphorylation.

\section{C26 CM reduced the expression of genes involved with ribosomal biogenesis, but DRP1 knockdown did not affect it}

Ribosomes are organelles consisting of ribonucleoprotein complexes, which catalyze the synthesis of muscle protein directed by mRNA (24). Ribosome biogenesis could be affected under various catabolic conditions, including sarcopenia, disuse atrophy, and denervation (24). Additionally, a similar phenomenon was also observed in a mouse model of cachexia (25). However, studies have shown that knockout of S6K1 (the 70-kDa ribosomal protein S6 kinase (S6K), also called $\left.\mathrm{p} 70^{\mathrm{S} 6 \mathrm{~K}}\right)$, a major regulator of protein synthesis at the ribosomal level, activate mitochondrial fission and DRP1 expression in both mouse embryo fibroblasts and Henrietta Lack (HeLa) cells $(26,27)$. Given the potential association between DRP1 and ribosomal biogenesis during muscle atrophy, as well as our transcriptome analysis, we examined the markers of ribosomal biogenesis at the mRNA level. The genes exhibited ribosomal DNA (rDNA)-related activity (Pol-I, c-Myc, and Ubf), ribosomal RNA (rRNA) processing (Nop56), and ribosome assembly ( $\mathrm{Ncl}$ ) (24). These outcomes indicated that the mRNA level of $\operatorname{Ubf}(\mathrm{P}=0.0449)$ in $C M$ group was reduced compared to that of the HS group, while DRP1 knockdown did not significantly abrogate this decrease (Figure 4). These results demonstrated a reduction in ribosomal activity during C26 CM-induced myotube atrophy.

\section{Discussion}

CAC-induced muscle atrophy leads to a drastic effect on cancer treatment, quality of life, and patient survival. However, the exact mechanism is unclear and effective treatments remain elusive. In this study, we incubated C2C12 myotubes with CM from C26 colon cancer cells and generated a model of induced-muscle atrophy to simulate cachexia in vitro. Along with myotube atrophy, we also observed an activation of DRP1. To explore its role further, we used lentivirus as a vector to regulate its expression. Interestingly, our findings indicated that DRP1 overexpression in myotubes exacerbated myotube atrophy, while DRP1 knockdown markedly ameliorated this phenomenon. Further transcriptome analysis in the DRP1 knockdown group suggested a significant activation of ribosomal biogenesis and oxidative phosphorylation. However, for verification, although C26 CM induced an increased expression of $U b f$ (a marker of ribosomal biogenesis) at the mRNA level, the knockdown of DRP1 expression did not alter its level.

White et al. and Brown et al. revealed up-regulation of mitochondrial fission regulators (mainly FIS1) and downregulation of fusion regulators (mainly MFN1/MFN2) in a cachexia model of $\mathrm{Apc}^{\mathrm{Min} / \mathrm{t}}$ mice $(11,12,16)$. Moreover, Fix et al. reported that incubation of $\mathrm{C} 2 \mathrm{C} 12$ myotubes with interleukin-6 (IL-6), a major cytokine participates in various CAC conditions, also appeared to induce DRP1 and FIS1 expression $(11,28)$. These results were in line with our major finding, namely that DRP1 was activated under cachexia. Also, Romanello et al. showed, in a mouse model of fasting, that the dominant-negative mutation of DRP1 in skeletal muscle alleviates the corresponding atrophy. In contrast, transduction with a DRP1 overexpression vector could intensify muscle wasting (10). On the other hand, Touvier et al. reported that overexpression of muscle-specific DRP1 in mice manifested as a reduction of muscle mass, exercise performance, postnatal muscle growth, as well as impaired mitochondrial network remodeling (29). These findings were consistent with ours; altering mitochondrial fission by influencing its main regulator could affect muscle mass.

However, other recent studies have proposed a different perspective. Favaro et al. reported that muscle-specific 
DRP1 knockout led to a significant muscle atrophy and degeneration in mice (30). Nevertheless, a follow-up mouse study from the same laboratory suggested that DRP1 knockdown reversed the impairment induced by OPA1 knockout in skeletal muscle (31). Also, Moore et al. generated a muscle-specific DRP1 heterozygote mice mouse (with a $40 \%$ reduction). In this model, the mice exhibited decreased endurance and running performance, as well as reduced adaptation to exercise training, although no muscle atrophy was observed (32). In our study, the siRNA vector used in DRP1 knockdown had a higher efficiency $(68 \%)$ and this extra increase did not impair the biology and differentiation of $\mathrm{C} 2 \mathrm{C} 12$ myotubes, which was also confirmed by Jheng et al. (33). We speculated this gap (28\%) was probably compensated by the enhancement under cachexia. Altogether, these outcomes demonstrated that DRP1, as a vital factor involved in mitochondrial fission, plays an essential role in skeletal muscle turnover and function.

The equilibrium of mitochondrial dynamics is essential to counteract the accumulation of defective mitochondrial components, leading to rapid morphological adaptations, as well as functional recovery. Under pathological conditions, excessive fission produces dysfunctional mitochondria, consuming quantities of adenosine triphosphate (ATP) to maintain membrane potential with a low efficiency of ATP production (6). It also interferes with the balance of mitochondrial dynamics and compromises mitochondrial function, and even impairs cell health. However, reversing this imbalance results in a significant recovery, specifically in diseases associated with abnormal mitochondrial networks (34-37). Thus, the balance between fusion and fission states has been considered as a critical event for muscle mass and whole-body homeostasis. In particular, we supposed that during cachexia, knockdown of DRP1 reversed the excess of the fission state to a certain extent, and thereby disrupted atrophy in our study. Inversely, overexpression of DRP1 intensified the degree of imbalance and its concomitant effects. All of these outcomes directly demonstrated the relationship between DRP1 levels and muscle atrophy under cachexia.

In the process of CAC, skeletal muscle atrophy is promoted by various factors. These factors include reduced nutrition intake and physical activity, as well as substantial metabolic alterations induced by tumor-secreted factors and inflammatory cytokines (3).

To our knowledge, pathways activated by cytokines massively contribute to skeletal muscle atrophy. These cytokines are produced by tumor and stromal cells in tumor microenvironment, as well as by cells from the immune system. The factors then activate ubiquitin-proteasome and autophagy systems. In general, activation of the systems induces a specific degradation of myofibrillar proteins that construct sarcomeres and supplement contractile function to skeletal muscle. Loss of the myofibrillar proteins leads to muscle atrophy and weakness. In addition, transforming growth factor- $\beta$ (TGF $\beta$ ) could alter $\mathrm{Ca}^{2+}$ handling, resulting in the dysfunction of sarcomere without the loss of sarcomeric proteins. Moreover, insulin-like growth factor I (IGF-I) pathway through RAC serine/threonineprotein kinase (AKT) could mediate functional blunt of the transcription factors forkhead box protein O1 (FOXO1) and/or FOXO3. And this signal inhibits the transcription of atrophy genes with the blockade of the nuclear translocation and their overall expressions. However, this inhibitory activity is frequently repressed in cachexia, resulting in an activation of E3 ubiquitin ligases and autophagy system (3).

Alternatively, impaired muscle regeneration is observed in CAC. In mice bearing C26 tumor, Guttridge et al. detected an upregulation of Pax7 expression and Pax7+ cells in muscle. The increased Pax7 indicated an activation of satellite cell (SC) under tumor-derived stimuli. But persistent activation of SC could not restore muscle regeneration but augment muscle atrophy. Further, reduction of $\mathrm{Pax} 7$ or overexpression of its downstream target, MyoD, restored muscle differentiation and protected muscle from loss (38). Hogan et al. observed that one of tumor-derived factor, CXCL1, potently antagonized muscle regeneration and interfered with muscle SC homeostasis in vivo. Moreover, CXCL1 triggered a robust and specific neutrophil/M2 macrophage response that likely exacerbates muscle regeneration defects (39). On the other hand, because of metabolic alterations of substrate utilization in cachectic patients, it was supposed that SC sensed and responded to these systemic metabolic changes. Ryall et al. showed that MyoD expression was modulated by metabolism-induced epigenetic changes, establishing a dependence of myogenic regulatory factor (MRF) expression on the metabolic state of SC (40).

Ribosomal biogenesis, which is involved in ribosomal RNA (rRNA) transcription and mRNA translation, modulates the content of ribosomes and ultimately regulates the protein synthesis. A widely accepted view was that the enhanced ribosomal biogenesis appears to be more strongly associated with skeletal muscle hypertrophy by resistance exercise and overload (41). However, our transcriptome 
analysis revealed that DRP1 knockdown activated the ribosomal biogenesis pathway, which assists in restoring protein synthesis and led to alleviated myotube atrophy. Concerning the role of ribosomes in muscle atrophy, White et al. reported a reduced phosphorylation of S6K (a major marker of ribosomal biogenesis) in mice with cachexia of different degrees (25). Correspondingly, Williams et al. pointed out that intravenous amino acid supplements resulted in an increased S6K phosphorylation, which was more pronounced among patients with CAC compared to those without CAC (42). Furthermore, in a model of glucocorticoid-induced muscle atrophy in rats, Schakman et al. found that S6K phosphorylation was repressed (43). These evidences implicate a significant association between ribosomal biogenesis and catabolic conditions, and support our findings that ribosomal biogenesis is a novel mechanism that alleviates DRP1 knockdown-induced muscle atrophy.

Furthermore, these outcomes also indicated an involvement of oxidative phosphorylation activity. The reduction of oxidative phosphorylation is prominent during metabolic syndromes induced by some chronic diseases, and increases the release of some proinflammatory cytokines (44). Heart is an organ with a high energetic demand. Mutations in nuclear DNA (nDNA) coded mitochondrial genes and mitochondrial DNA (mtDNA) mutations which causes partial OXPHOS defects, could generate cardiomyopathy. In addition, accumulation of somatic mtDNA mutations, along with effects of secondary inflammation from the release of mitochondrial damage associated molecular patterns (DAMPs), induce the mitochondrial dysfunction. And this process would exacerbate cardiomyopathy as well (45). Moreover, defective oxidative phosphorylation has also been considered a major cause of skeletal muscle complications in aging (46). In some specific conditions of muscle atrophy, such as denervation and immobilization, there was an activation of tumor necrosis factor-like weak inducer of apoptosis/ fibroblast growth factor-inducible 14 (TWEAK/Fn14) signaling, which led to a significant repression of oxidative phosphorylation capacity (47). However, a recent study by Johnston $e t$ al. verified the direct relationship between TWEAK/Fn 14 and muscle atrophy in a mouse model of cachexia (48). These results implied that oxidative phosphorylation plays an important role in relevant muscle atrophy, which was consistent with our data. Interestingly, the pathways of Parkinson's disease, Alzheimer's disease, and Huntington's disease were also partly altered (data not shown). This phenomenon could be probably attributed to the intimate relationship of these diseases and mitochondrial fission, especially DRP1 activity (49,50).

In this model, the mRNA of $U b f$, which acts to enhance ribosomal DNA (rDNA) promoter events (51) during transcription, was downregulated. Since the markers were not comprehensive to represent the status of ribosomal biogenesis, we did not observe a significant alteration from DRP1 knockdown. This might be attributed to the deficiency of the samples $(n=4)$. However, transcriptome analysis between the KD and Dc groups, and the KD and CM groups (data not shown) both exhibited a prominent ribosomal activation. Thus, we still consider ribosomal biogenesis as a novel mechanism involved in this model. Nevertheless, the influence of ribosomal biogenesis under different conditions of cachexia requires further examination.

A limitation of the study is lack of experiments in vivo. The exact mechanism of muscle atrophy is complicated, considering effect of the immune system and tumor microenvironment which will also contribute to this process. Therefore, more researches in vivo on the issue are required.

\section{Conclusions}

Our model guaranteed an increase in DRP1 levels during cachexia in vitro, and the regulation of DRP1 could modulate the atrophy in this model. Specifically, DPR1 knockdown reduced myotube atrophy, which was associated with increased ribosomal biogenesis. Thus, our findings indicate that DRP1 plays an important role in C26-related $\mathrm{CAC}$, and is implicated as a potential therapeutic target in cachexia. Nonetheless, the role of DRP1 in different CAC conditions is not fully understood, and the character of mitochondrial dynamics in muscle protein turnover remains unclear. Therefore further studies are warranted.

\section{Acknowledgments}

We would like to thank Charlesworth Author Service for assistance in writing this manuscript; Genechem, Shanghai, China for lentivirus synthesis of si-RNA and express DRP1; and Genewiz, Suzhou, China for transcriptome sequencing. Funding: None.

\section{Footnote}

Reporting Checklist: The authors have completed the 
MDAR reporting checklist. Available at https://dx.doi. org/10.21037/tcr-21-751

Data Sharing Statement: Available at https://dx.doi. org/10.21037/tcr-21-751

Conflicts of Interest: All authors have completed the ICMJE uniform disclosure form (available at https://dx.doi. org/10.21037/tcr-21-751). The authors declare that vectors with siRNAs targeting DRP1 and a negative control siRNA were obtained from GeneChem Inc. (Shanghai, China), and the processes of sequencing and analysis were carried out by GENEWIZ (Suzhou, China); there is no professional or other personal interest in services from Genechem, Shanghai, China and Genewiz, Suzhou, China that could be construed as influencing the position presented in the manuscript. The authors have no other conflicts of interest to declare.

Ethical Statement: The authors are accountable for all aspects of the work in ensuring that questions related to the accuracy or integrity of any part of the work are appropriately investigated and resolved.

Open Access Statement: This is an Open Access article distributed in accordance with the Creative Commons Attribution-NonCommercial-NoDerivs 4.0 International License (CC BY-NC-ND 4.0), which permits the noncommercial replication and distribution of the article with the strict proviso that no changes or edits are made and the original work is properly cited (including links to both the formal publication through the relevant DOI and the license). See: https://creativecommons.org/licenses/by-nc-nd/4.0/.

\section{References}

1. Pressoir M, Desne S, Berchery D, et al. Prevalence, risk factors and clinical implications of malnutrition in French Comprehensive Cancer Centres. Br J Cancer 2010;102:966-71.

2. Hebuterne X, Lemarie E, Michallet M, et al. Prevalence of malnutrition and current use of nutrition support in patients with cancer. JPEN J Parenter Enteral Nutr 2014;38:196-204.

3. Baracos VE, Martin L, Korc M, et al. Cancer-associated cachexia. Nat Rev Dis Primers 2018;4:17105.

4. Fearon K, Strasser F, Anker SD, et al. Definition and classification of cancer cachexia: an international consensus. Lancet Oncol 2011;12:489-95.

5. Martin L, Birdsell L, Macdonald N, et al. Cancer cachexia in the age of obesity: skeletal muscle depletion is a powerful prognostic factor, independent of body mass index. J Clin Oncol 2013;31:1539-47.

6. Romanello V, Sandri M. The connection between the dynamic remodeling of the mitochondrial network and the regulation of muscle mass. Cell Mol Life Sci 2021;78:1305-28.

7. Tilokani L, Nagashima S, Paupe V, et al. Mitochondrial dynamics: overview of molecular mechanisms. Essays Biochem 2018;62:341-60.

8. Taguchi N, Ishihara N, Jofuku A, et al. Mitotic phosphorylation of dynamin-related GTPase Drp1 participates in mitochondrial fission. J Biol Chem 2007;282:11521-9.

9. Romanello V, Sandri M. Mitochondrial Quality Control and Muscle Mass Maintenance. Front Physiol 2016;6:422.

10. Romanello V, Guadagnin E, Gomes L, et al. Mitochondrial fission and remodelling contributes to muscle atrophy. EMBO J 2010;29:1774-85.

11. White JP, Puppa MJ, Sato S, et al. IL-6 regulation on skeletal muscle mitochondrial remodeling during cancer cachexia in the ApcMin/+ mouse. Skelet Muscle 2012;2:14.

12. White JP, Baltgalvis KA, Puppa MJ, et al. Muscle oxidative capacity during IL-6-dependent cancer cachexia. Am J Physiol Regul Integr Comp Physiol 2011;300:R201-11.

13. Marzetti E, Lorenzi M, Landi F, et al. Altered mitochondrial quality control signaling in muscle of old gastric cancer patients with cachexia. Exp Gerontol 2017;87:92-9.

14. Sebastian D, Sorianello E, Segales J, et al. Mfn2 deficiency links age-related sarcopenia and impaired autophagy to activation of an adaptive mitophagy pathway. EMBO J 2016;35:1677-93.

15. Barreto R, Mandili G, Witzmann FA, et al. Cancer and Chemotherapy Contribute to Muscle Loss by Activating Common Signaling Pathways. Front Physiol 2016;7:472.

16. Brown JL, Rosa-Caldwell ME, Lee DE, et al. Mitochondrial degeneration precedes the development of muscle atrophy in progression of cancer cachexia in tumour-bearing mice. J Cachexia Sarcopenia Muscle 2017;8:926-38.

17. Ishihara N, Nomura M, Jofuku A, et al. Mitochondrial fission factor Drp1 is essential for embryonic development and synapse formation in mice. Nat Cell Biol 2009;11:958-66.

18. Wakabayashi J, Zhang Z, Wakabayashi N, et al. 
The dynamin-related GTPase Drp1 is required for embryonic and brain development in mice. J Cell Biol 2009; 186:805-16.

19. Kim B, Kim JS, Yoon Y, et al. Inhibition of Drp1dependent mitochondrial division impairs myogenic differentiation. Am J Physiol Regul Integr Comp Physiol 2013;305:R927-38.

20. Seto DN, Kandarian SC, Jackman RW. A Key Role for Leukemia Inhibitory Factor in C26 Cancer Cachexia. J Biol Chem 2015;290:19976-86.

21. Pigna E, Berardi E, Aulino P, et al. Aerobic Exercise and Pharmacological Treatments Counteract Cachexia by Modulating Autophagy in Colon Cancer. Sci Rep 2016;6:26991.

22. Liu D, Qiao X, Ge Z, et al. IMB0901 inhibits muscle atrophy induced by cancer cachexia through MSTN signaling pathway. Skelet Muscle 2019;9:8

23. Sin J, Andres AM, Taylor DJ, et al. Mitophagy is required for mitochondrial biogenesis and myogenic differentiation of C2C12 myoblasts. Autophagy 2016;12:369-80.

24. Chaillou T, Kirby TJ, McCarthy JJ. Ribosome biogenesis: emerging evidence for a central role in the regulation of skeletal muscle mass. J Cell Physiol 2014;229:1584-94.

25. White JP, Baynes JW, Welle SL, et al. The regulation of skeletal muscle protein turnover during the progression of cancer cachexia in the Apc(Min/+) mouse. PLoS One 2011;6:e24650.

26. Tran Q, Jung JH, Park J, et al. S6 kinase 1 plays a key role in mitochondrial morphology and cellular energy flow. Cell Signal 2018;48:13-24.

27. Park J, Tran Q, Mun K, et al. Involvement of S6K1 in mitochondria function and structure in HeLa cells. Cell Signal 2016;28:1904-15.

28. Fix DK, VanderVeen BN, Counts BR, et al. Regulation of Skeletal Muscle DRP-1 and FIS-1 Protein Expression by IL-6 Signaling. Oxid Med Cell Longev 2019;2019:8908457.

29. Touvier T, De Palma C, Rigamonti E, et al. Muscle-specific Drp1 overexpression impairs skeletal muscle growth via translational attenuation. Cell Death Dis 2015;6:e1663.

30. Favaro G, Romanello V, Varanita T, et al. DRP1-mediated mitochondrial shape controls calcium homeostasis and muscle mass. Nat Commun 2019;10:2576.

31. Romanello V, Scalabrin M, Albiero M, et al. Inhibition of the Fission Machinery Mitigates OPA1 Impairment in Adult Skeletal Muscles. Cells 2019;8:597.

32. Moore TM, Zhou Z, Cohn W, et al. The impact of exercise on mitochondrial dynamics and the role of Drp1 in exercise performance and training adaptations in skeletal muscle.
Mol Metab 2019;21:51-67.

33. Jheng HF, Tsai PJ, Guo SM, et al. Mitochondrial fission contributes to mitochondrial dysfunction and insulin resistance in skeletal muscle. Mol Cell Biol 2012;32:309-19.

34. D'Amico D, Mottis A, Potenza F, et al. The RNA-Binding Protein PUM2 Impairs Mitochondrial Dynamics and Mitophagy During Aging. Mol Cell 2019;73:775-87.e10.

35. Varanita T, Soriano ME, Romanello V, et al. The OPA1dependent mitochondrial cristae remodeling pathway controls atrophic, apoptotic, and ischemic tissue damage. Cell Metab 2015;21:834-44.

36. Civiletto G, Varanita T, Cerutti R, et al. Opa1 overexpression ameliorates the phenotype of two mitochondrial disease mouse models. Cell Metab 2015;21:845-54.

37. Rana A, Oliveira MP, Khamoui AV, et al. Promoting Drp1-mediated mitochondrial fission in midlife prolongs healthy lifespan of Drosophila melanogaster. Nat Commun 2017;8:448

38. He WA, Berardi E, Cardillo VM, et al. NFkappaB-mediated Pax7 dysregulation in the muscle microenvironment promotes cancer cachexia. J Clin Invest 2013;123:4821-35.

39. Hogan KA, Cho DS, Arneson PC, et al. Tumor-derived cytokines impair myogenesis and alter the skeletal muscle immune microenvironment. Cytokine 2018;107:9-17.

40. Ryall JG, Dell'Orso S, Derfoul A, et al. The NAD(+)dependent SIRT1 deacetylase translates a metabolic switch into regulatory epigenetics in skeletal muscle stem cells. Cell Stem Cell 2015;16:171-83.

41. Kim HG, Guo B, Nader GA. Regulation of Ribosome Biogenesis During Skeletal Muscle Hypertrophy. Exerc Sport Sci Rev 2019;47:91-7.

42. Williams JP, Phillips BE, Smith K, et al. Effect of tumor burden and subsequent surgical resection on skeletal muscle mass and protein turnover in colorectal cancer patients. Am J Clin Nutr 2012;96:1064-70.

43. Schakman O, Kalista S, Bertrand L, et al. Role of Akt/ GSK-3beta/beta-catenin transduction pathway in the muscle anti-atrophy action of insulin-like growth factor-I in glucocorticoid-treated rats. Endocrinology 2008;149:3900-8.

44. Egan B, Zierath JR. Exercise metabolism and the molecular regulation of skeletal muscle adaptation. Cell Metab 2013;17:162-84.

45. Murphy E, Ardehali H, Balaban RS, et al. Mitochondrial Function, Biology, and Role in Disease: A Scientific Statement From the American Heart Association. Circ Res 
2016;118:1960-91.

46. Peterson CM, Johannsen DL, Ravussin E. Skeletal muscle mitochondria and aging: a review. J Aging Res 2012;2012:194821.

47. Sato S, Ogura Y, Kumar A. TWEAK/Fn14 Signaling Axis Mediates Skeletal Muscle Atrophy and Metabolic Dysfunction. Front Immunol 2014;5:18.

48. Johnston AJ, Murphy KT, Jenkinson L, et al. Targeting of Fn14 Prevents Cancer-Induced Cachexia and Prolongs Survival. Cell 2015;162:1365-78.

Cite this article as: Mao X, Meng Q, Han J, Shen L, Sui X, Gu Y, Wu G. Regulation of dynamin-related protein 1 (DRP1) levels modulates myoblast atrophy induced by C26 colon cancer-conditioned medium. Transl Cancer Res 2021;10(6):3020-3032. doi: 10.21037/tcr-21-751
49. Oliver D, Reddy PH. Dynamics of DynaminRelated Protein 1 in Alzheimer's Disease and Other Neurodegenerative Diseases. Cells 2019;8:961.

50. Yan X, Wang B, Hu Y, et al. Abnormal Mitochondrial Quality Control in Neurodegenerative Diseases. Front Cell Neurosci 2020;14:138.

51. Lempiainen H, Shore D. Growth control and ribosome biogenesis. Curr Opin Cell Biol 2009;21:855-63.

(English Language Editor: A. Kassem) 